\title{
METÁFORAS DE LA MATERNIDAD EN UN SISTEMA DE ATENCIÓN SANITARIA DE LA INFANCIA EN CHILE: ENTRE LA NATURALEZA Y EL CAPITAL HUMANO
}

Metaphors of Motherhood in a Children's Healthcare System in Chile: Between Nature and Human Capital

Claudia Calquín-Donoso, ${ }^{1}$ Cristopher Yáñez-Urbina ${ }^{2}$

Correo electrónico: claudia.calquín@usach.cl

1. Escuela de Psicología, Facultad de Humanidades, Universidad de Santiago de Chile. (Santiago de Chile, Chile)

2. Centro de Desarrollo Cognitivo, Facultad de Educación, Universidad Diego Portales. (Santiago de Chile, Chile)

Recibido: 14/04/2020 Aceptado: 20/05/2020

(c) (i) () 


\section{RESUMEN}

El artículo presenta resultados parciales de una investigación mayor dirigida a conocer los significados circulantes de la maternidad, la infancia y su cuidado en un sistema de atención sanitaria en Chile. Para lograr este objetivo, se realizó un análisis de metáforas usando el procedimiento de identificación de metáforas (PMI) aplicado a un corpus textual conformado por materiales de lectura producidos y utilizados por el sistema. Los resultados indican que las diferentes formas a través de las cuales se representa el cuerpo materno, desde objetos inanimados hasta una práctica empresarial, permiten conocer una amplia gama de imaginarios, ambivalencias y demandas que se realizan a las prácticas maternas en el contexto sanitario y que configuran desigualdades de género. Concluimos que la maternidad sigue enclavada en el imaginario de la naturaleza, como una experiencia privada y hermética en la que la figura del binomio madre e hijo/a sigue siendo fundamental para los objetivos sanitarios al que se han sumado nuevas demandas vinculadas a la reproducción del capital humano. Esto bloquea formas alternativas de representar la maternidad, las negociaciones de las identidades maternas, otros deseos que habitan en las mujeres y las posiciones e intereses diferenciales entre niños/as y mujeres.

Palabras Claves: maternidad; cuidados de la infancia; metáforas; sistema Chile Crece Contigo.

\section{ABSTRACT}

This article presents the partial results of a larger investigation aimed at knowing the circulating meanings of motherhood, childhood and their care in a healthcare system in Chile. To achieve this aim, a metaphor analysis was performed using the metaphor identification procedure (MIP) applied to a textual corpus made up of reading materials produced and used by the system. The results indicate that the different forms through which the maternal body is represented, from inanimate objects to a business practice, allow knowing a wide range of imaginaries, ambivalences and demands that are made of maternal practices in the health context and that shape gender inequalities. We conclude that motherhood continues to be embedded in the imaginary of nature as a private and hermetic experience in which the figure of the mother-child binomial continues to be fundamental for the health objectives to which new demands linked to the reproduction of human capital have been added. This hinders alternative ways of representing motherhood, the negotiations of maternal identities, other desires that inhabit women, and the differential positions and interests between children and women.

Keywords: Maternity; Childhood Care; Metaphors; Chile Crece Contigo system. 


\section{INTRODUCCIÓN}

A partir de la década del 2000, Chile impulsó un proceso de reforma en salud que, junto con impulsar un sistema de Garantías Explícitas en Salud [GES] para garantizar por ley un conjunto de prestaciones sanitarias, cambió el foco, desde una atención fuertemente sectorizada hacia la implementación de sistemas integrales de atención con énfasis en la promoción de estilos de vida saludables y la prevención de enfermedades no contagiosas. En el marco de estas reformas, en el año 2006 se crea el Subsistema de Protección Integral de la Infancia Chile Crece Contigo [CCC] dirigido a asegurar un acceso expedito a servicios y prestaciones que atiendan a las necesidades específicas de las infancias en riesgo y más vulnerables, especialmente aquellas que presentan el diagnóstico de rezago o retraso en el desarrollo.

Hablamos de un sistema intersectorial liderado por el Ministerio de Salud y que a nivel de implementación — desde el control prenatal hasta el apoyo profesional en la estimulación temprana para niños y niñas hasta los 4 años — se apoya fuertemente en el sistema de cuidados familiares próximos al entorno maternal, erigiendo de manera especial a las mujeres/madres como proveedoras principales de cuidados, superación de la vulnerabilidad y promoción del desarrollo de los niños y las familias (Castillo, 2016; Magaña et al., 2011; Paura y Zibbechi, 2018).

En este contexto, en el presente artículo reportamos algunos resultados parciales de un proyecto de investigación más amplio que indaga, desde una perspectiva crítica, los discursos sobre la infancia y el cuidado materno presentes en el CCC. De forma puntual, realizamos un análisis cualitativo de metáforas recurrentes en una serie de documentos producidos en el subsistema y dirigidos a profesionales de la salud. Nuestro interés es mostrar las formas en que se construyen algunos supuestos de las familias y las mujeres destinatarias de la acción pública.

Argumentamos que, las metáforas, más que un recurso retórico, son un recurso analizador para captar los imaginarios sobre la maternidad y el cuidado de la infancia que habitan en las políticas de salud infantil como en los saberes expertos, y que nos permite acceder a connotaciones y evocaciones que una particular visión del mundo pone en juego para construir realidades compartidas. Con esto, queremos aportar tanto a los estudios sociales de la salud como a la misma política pública, en la medida en que las 
representaciones simbólicas juegan un papel crucial en la configuración de las desigualdades de género y los roles de las mujeres asignados en las políticas de estado.

\section{EL CONTEXTO DE INVESTIGACIÓN: EL SISTEMA CHILE CRECE CONTIGO}

El CCC es un complejo sistema intersectorial de intervención sociosanitaria centrado en el desarrollo biopsicosocial infantil. Forma parte de lo que, en política pública, se ha llamado la «generación Bachelet» (Caro, 2009) y que permitió dar cohesión y contenido a la idea de un gobierno orientado a un Estado de protección social (Garretón, 2012) con el objetivo de fortalecer la asistencia social y sanitaria hacia mujeres y niños/as en contextos de riesgo sanitario y social, otorgándole a estas prestaciones el carácter de garantía constitucional de derechos (Hardy, 2011).

Desde su creación, el CCC se propuso como una respuesta gubernamental a los altos porcentajes de pobreza infantil existente en Chile y, si bien representó un avance en materia de garantías y acceso a derechos sociales y de salud —a través de un piso mínimo de bienestar garantizados estatalmente- , lo cierto es que la fuerte desigualdad social y de género, y sus condiciones estructurales de producción fueron escasamente abordados en sus objetivos programáticos (Solimano, 2018). Así, el camino para superar la vulnerabilidad y los riesgos en salud infantil se realiza a partir de una matriz neoliberal (Garretón, 2012; Solimano, 2018) que, por un lado, focaliza la acción del estado en las poblaciones más pauperizadas $\mathrm{y}$, por otro, despliega intervenciones altamente individualizadas bajo un esquema de la clásica división sexual del trabajo (Magaña, et al. 2011). Vemos, por lo tanto, que el sistema de atención se articula en un modelo que se basa en la responsabilidad individual y familiar de la gestión de los riesgos y la provisión de bienes y servicios básicos, en que las mujeres madres siguen siendo los principales sujetos de la provisión de bienestar al mismo tiempo que se las describe como carentes de capacidades y estrategias de afrontamiento para manejar los riesgos (Arteaga e Íñigo, 2015; Fuentes y Brembeck, 2019).

Así, el sistema captura a las poblaciones más pauperizadas a una forma de intervención que responden a una perspectiva de los modelos de «competencias parentales» (Contreras et al., 2015) introduciendo, además, los hallazgos de la neurociencia y sus conceptos de plasticidad neuronal y periodos críticos y las 
ampliamente difundidas «intervenciones en apego» (Castillo, 2016). Apreciamos que el sistema desarrolla un fuerte discurso que promueve la integración social de las familias más pobres —usando el sintagma «equidad desde la gestación»— bajo la forma de problemas individuales, psicológicos y trabajando en la economía personal de las familias (Castel, 2010) en la que los límites entre responsabilización y culpabilización se vuelven porosos.

\section{APUNTES METODOLÓGICOS: EL SISTEMA CHILE CRECE CONTIGO COMO OPERADOR SEMIÓTICO}

Definimos el CCC como un operador semiótico que produce aquello que dice representar (Austin, 1991). De esta forma, el análisis cualitativo y la perspectiva pragmática (Escandell, 1993) nos permitieron acceder al conjunto de significados que operan en esta política pública. Así, el amplio material documental que la conforman —artículos académicos, manuales de intervención, hojas de rutas, material educativo, normas técnicas y pautas de detección, entre otros documentos - fueron agrupados, ordenados y clasificados en un corpus documental que dio por resultado la selección de 11 documentos según criterios de relevancia, que pertenecieran a documentos de esta política y pertinencia, que contuvieran contenidos que aportaran al objetivo de la investigación (Ver Tabla núm. 1)

En primer lugar, a estos documentos se les aplicó un análisis de contenido temático (Cárcamo, 2016) en el que se establecieron las unidades de significados más relevantes para los objetivos de la investigación (maternidad, infancia, intervención, comunidad, sociedad y estado). En un segundo momento, sobre estas unidades de significado se identificaron diversas prácticas discursivas que permitieran indagar de forma más profunda en los modos en que, por un lado, el sistema construye sus objetos, sujetos y problemas y; por otro, las visiones del mundo que lo habitan. De las múltiples estrategias, presentamos las que corresponden a los usos metafóricos del lenguaje que, lejos de ser un elemento ornamental del discurso, modelan nuestra percepción, nuestro pensamiento y nuestras acciones. 
Tabla 1. Corpus documental a analizar

\begin{tabular}{|c|c|c|c|}
\hline \multirow[t]{2}{*}{$\mathbf{N}$} & \multirow{2}{*}{\multicolumn{3}{|c|}{$\begin{array}{l}\text { Publicación o } \\
\text { Locución }\end{array}$}} \\
\hline & & & \\
\hline 1 & $\begin{array}{l}\text { Ley } 20.379 \text {, que crea el sistema intersectorial de } \\
\text { protección social e institucionaliza el subsistema de } \\
\text { protección integral a la infancia «Chile crece contigo» }\end{array}$ & 2009 & D 1 \\
\hline 2 & $\begin{array}{l}\text { Manual para el apoyo y el seguimiento del desarrollo } \\
\text { psicosocial de los niños y niñas de } 0 \text { a } 6 \text { años }\end{array}$ & 2008 & D 2 \\
\hline 4 & $\begin{array}{l}\text { Manual de orientaciones técnicas para las modalidades } \\
\text { de apoyo del desarrollo infantil: guía para equipos } \\
\text { locales }\end{array}$ & 2013 & D 4 \\
\hline 5 & Cuando Copiar es Bueno (V Edición) & 2016 & D 5 \\
\hline 6 & $\begin{array}{l}\text { Discurso S. E. Michel Bachelet Conmemoración } 10 \\
\text { años de Chile Crece Contigo }\end{array}$ & 2017 & D 6 \\
\hline 7 & $\begin{array}{l}\text { Intervenciones psicológicas perinatales en depresión } \\
\text { materna y vínculo madre-bebé: una revisión sistemática }\end{array}$ & & D7 \\
\hline 8 & $\begin{array}{l}\text { Interacciones tempranas y género infantil en familias } \\
\text { monoparentales chilenas }\end{array}$ & 2012 & D8 \\
\hline 9 & Bienestar y apego en la sala cuna Módulo 1 & 2007 & D9 \\
\hline 10 & $\begin{array}{l}\text { Apego: representaciones de educadoras de párvulos en } \\
\text { jardines infantiles en Temuco, Chile }\end{array}$ & 2016 & D10 \\
\hline 11 & $\begin{array}{l}\text { El futuro de los niños es siempre hoy. Propuestas del } \\
\text { Consejo Asesor Presidencial para la Reforma de las } \\
\text { Políticas de Infancia }\end{array}$ & 2006 & D11 \\
\hline
\end{tabular}

Fuente: Elaboración propia

Al decir de Lizcano (2006), no somos nosotras quienes las decimos, son ellas las que nos dicen y dicen el mundo. El análisis de las metáforas permite responder a preguntas como: ¿Qué prácticas posibilita cada una de estas metáforas?, ¿qué espacio discursivo construyen?, ¿para quién están disponibles?, ¿qué rasgos ocultan o destacan? (Casado, 1999). El procedimiento usado correspondió al «procedimiento para la identificación de metáforas» (MIP) (Pragglejaz Group, 2007), que consiste en las siguientes fases: 
1. Determinar las unidades léxicas del texto (luego de una lectura exhaustiva para comprender su significado general).

2. Identificar el significado contextual de cada unidad léxica, esto es, el significado que esta unidad tiene en la situación en la que se está usando. Para esto se recurre a un diccionario.

3. Verificar si hay un significado más básico de esta unidad léxica e identificarlo. Este significado se caracteriza por ser más concreto, porque lo que evoca es más fácil de imaginar, ver, escuchar, sentir, oler y gustar, por estar relacionado con la acción corporal y por ser más específico.

4. Determinar si el significado más básico es lo suficientemente distinto del significado contextual.

5. Examinar si el significado contextual puede relacionarse con el básico mediante alguna forma de similitud.

Tomando la recomendación de González (2014), nos centramos en aquellas expresiones metafóricas que no son simples modulaciones estilísticas u ornamentales ocasionales sino metáforas conceptuales constitutivas, es decir, en aquellas dan forma a la estructura conceptual del texto. En ese sentido, la segunda fase, el análisis contextual, tal como apunta la perspectiva pragmática, fue central, pues permitió establecer relaciones entre las metáforas, el contexto discursivo en el que se generan y la función que adquieren en la política y para organizar los significados que nos interesaba relevar. De ahí que, a diferencia del modelo de González, el criterio de selección de las metáforas a analizar no fue de orden cuantitativo, sino ilocucionario en el sentido de que se presentaron expresiones metafóricas no frecuentes, pero si tenían la fuerza para constituir repertorios sobre la maternidad y el cuidado de los niños/as; es decir, lo que importó es que estas participaran de cierta organización del discurso, por lo que el trabajo interpretativo fue central en todo el proceso analítico.

Una vez identificadas y ordenadas las expresiones metafóricas, se procedió a categorizarlas de acuerdo a la teoría de la metáfora conceptual (Lakoff y Johnson, 1998), según la cual un área semántica o dominio se representa conceptualmente en términos de otro, denominados «dominio meta» (aquel dominio que queremos metaforizar) y «dominio fuente» (la imagen de donde extrajimos la metáfora), lo que nos facilitó realizar 
un conjunto de inferencias sobre los sistemas conceptuales que organizan el discurso sobre la maternidad.

\section{RESULTADOS}

En nuestro corpus, nos encontramos con expresiones metafóricas que configuran formas de identidad y de ser sujetos, relaciones y fenómenos. Identificamos las siguientes analogías presentes en las categorías emergentes maternidad e infancia: 1. El cuerpo de las mujeres como contenedor; 2. El cuerpo del niño/a como un libro; 3. La relación madrehijo como una relación animal; 4. La familia y la maternidad como una empresa.

Si ponemos atención en las siguientes expresiones: «En esta línea, los estudios describen secuelas biológicas, psicológicas y sociales de la depresión perinatal en la díada» (D7) y «Derivación al control de la díada antes de los 7 a 10 días postparto en la atención primaria de salud»(D4), vemos que lo que podría parecer un conjunto de expresiones e imágenes metafóricas aisladas o aleatorias, se revela como un sistema conceptual coherente para caracterizar el campo de la maternidad en que la figura del binomio madre/hijo es fundamental. Se trata de una particular forma de construcción discursiva que remite a la metáfora conceptual del «contenedor» o «recipiente» descrita como una metáfora ontológica (Lakoff y Johnson, 1998), que consiste en una distinción de límites entre un interior y un exterior, entre un adentro y un afuera. En el primer extracto la palabra clave, es la preposición «en» la que indica «el lugar donde está algo; especialmente si está en el interior de algo» (RAE, 2020). Por otro lado, hay un uso extensivo del sustantivo «gestante» para referirse a las mujeres usuarias, y que si bien no tiene un uso metafórico propiamente tal (no se trata de una sustitución), sí es un tropo constitutivo del lenguaje de la política de salud, en tanto la gestante es el sujeto de intervención, control y cuidado. «Gestante» lo entendemos como una sinécdoque pues reemplaza el significante más amplio —-mujer o usuaria — por un elemento en particular, como es la capacidad de reproducción; tomando la parte por el todo.

Vemos así que el sujeto femenino a partir de estas construcciones trópicas se construye como un parte de la díada (metáfora) y, por otro, como un ser exclusivamente reproductivo (sinécdoque) construyendo una imagen de las mujeres anclada estrictamente en su rol de madre, a tal punto que, por ejemplo, la salud mental de las mujeres se considera en estricta relación a la salud del niño y no hay ninguna referencia a la 
sexualidad de las mujeres, en la forma de un fenómeno sin discurso (Callejo, 2019). Estas figuras retóricas, lejos de ser diferentes en naturaleza, ambas tienden a solaparse una a otra contribuyendo a reafirmar la imagen más extendida de la maternidad. Se evoca la idea convencional de la mujer como incompleta o como vacía y que solo puede ser llenada o completada a través de la gestación (Imaz, 2001). La díada, además, representa la idealización de la maternidad al tomar la forma de una ilimitada pasión de la madre hacia el hijo y como una relación mediata, fusionada y de completa unidad.

Así como la relación de las metáforas con otros tropos es constitutiva de la significación, las metáforas no operan de forma aislada (Lakoff y Johnson, 1998) y sus significados se refuerzan gracias a sus relaciones con otras metáforas. Encontramos, de este modo, un segundo conjunto de expresiones metafóricas muy vinculadas a la anterior que evocan la «lectura» de forma explícita y al «libro» de manera implícita. Se usa de forma preferente para hablar de los procesos de comunicación y relación entre la madre y el bebé, estableciendo una analogía entre cuerpo y libro. Leemos las siguientes expresiones metafóricas: «Madres que no logran leer e interpretar adecuadamente las señales de sus hijos(as) y actuar de manera consistente para acoger sus necesidades» (D8). El significado contextual de estas expresiones emerge de la incorporación en la intervención en salud de las emociones y la comunicación familiar bajo lo que se llama el desarrollo de la «sensiblidad parental» o el «cuidado sensible». ${ }^{1}$ Si pensamos que el funcionamiento emocional no posee una estructura conceptual claramente definida (Lakoff y Johnson, 1998), el libro y la lectura permiten establecer relaciones entre un campo abstracto y un objeto concreto. De acuerdo a Vandendorpe (1999), el término «leer» que tiene su origen en el latín legere — recoger — está a asociado a la acción de espigar en la superficie de un campo y, según el DRAE (2020), se define como: 1. «pasar la vista por lo escrito o impreso comprendiendo la significación de los caracteres empleados» y 2. «comprender el sentido de cualquier tipo de representación gráfica». Considerando estas definiciones, hablamos de una metáfora sedimentada, que vincula de forma estrecha a la madre y sus criaturas, y que se revela por medio de la decodificación de los estados prelingüísticos del bebé. En consecuencia, el cuerpo del niño se construye como un libro y la madre, como una hermeneuta.

1 Se define como una habilidad de las madres «para percibir las señales y comunicaciones implícitas de la conducta del infante, interpretarlas acertadamente y, en base a ello, responder de un modo apropiado y oportuno». 
Si bien la metáfora del libro podría conducirnos a diversas formas de representar la lectura, como, por ejemplo, la lectura de un libro abierto, incompleto y en relación performativa con un lector no preformado o una práctica, que tal como señala su definición, tiene que ver con las palabras y los signos gráficos, su uso y la función que toma en los textos se realiza bajo una connotación naturalística y determinista —inclusive sancionadora. Este cuerpo-libro estaría escrito en el lenguaje de una naturaleza presimbólica, es decir, por cuasi signos. Por lo que se podría definir como una metáfora de «interacción no simbólica» (Blumer, 1982) en la que la madre debe responder certeramente a las señales corporales del bebé, bloqueando los posibles conflictos de interpretación propios de cualquier acto semiótico. Esta metáfora refuerza la figura de la «diada», introduciendo ideales del yo que estructuran, en cierta medida, las normas de género asociadas a una buena y a una mala maternidad bajo adjetivos como hábil, competente o sensible, activando o sugiriendo métodos de lectura apropiados. A través de ella se justifican distinciones binarias y altamente normalizadas, ya sea entre sujetas que leen o leen correctamente de las que no leen o leen incorrectamente, siendo las segundas, en ambos casos, posicionadas en la patología, lo indeseable inclusive en el reproche moral — como se alcanza a vislumbrar en el extracto «leen las señales del niño(a) desde el punto de vista del niño(a) y no desde el deseo del adulto» (D4)

Otro conjunto de metáforas son las expresiones que recurren a los «animales» para proyectar sobre las relaciones familiares algunas características de especies como aves y otros mamíferos no humanos. Se trata de una metáfora bidireccional en tanto es común la representación del parentesco animal en términos humanos. Es muy significativo el extracto siguiente, que revela una forma precisa de pensar el hogar y las instituciones de cuidado infantil: «El ingreso de un niño o niña a la sala cuna, debiera consistir en el tránsito armónico de un nido a otro nido» (D9). De acuerdo al DRAE (2020), nido es definido como: «Refugio que construyen las aves con hierbas, ramas, plumas u otros materiales blandos para poner sus huevos y albergar a sus crías». Este uso del hogar e inclusive de la sala cuna, como nido, tropos recurrentes en salud — por ejemplo, el «síndrome de nido vacío»— remite a una imagen animalizada del ser humano y romántica de los niños y el hogar; una sensación protectora y armónica que bloquea las asimetrías de poder. Es evidente que tras esta metáfora los niños/as y las mujeres están más próximas a la naturaleza que a la cultura activando procesos de subalternización dentro de una lógica evolutiva. 
Finalmente, la metáfora «empresarial», que se deriva de expresiones como competencias parentales, desempeño parental, lactancia exitosa, cultura del éxito, etc., inunda los textos. Leemos: «La conexión entre un intenso cuidado materno y la cultura del éxito parece ser intuitivamente correcta» (D10), «el desarrollo social de nuestro país depende crucialmente de cómo apoyemos a nuestros niños y niñas en sus etapas tempranas» (D11). Se trataría de pensar la maternidad esta vez desde un registro distinto al natural pero complementario, resultado de la incorporación de la teoría del capital humano y sus repertorios discursivos. La crianza se representa como una inversión a futuro y a la familia como una empresa en su doble acepción: como tarea, actividad, y como unidad de organización dedicada a actividades que generan ganancias y los niños/as como capital futuro. Se implementa una estrategia de intervención que plantea supuestos ideológicos concretos sobre la estructura y la dinámica de la vida familiar. Así, el cuerpo materno, además de lugar idóneo de prevención del riesgo por medio del control (Imaz, 2001), deviene lugar de proyección de ideologías económicas neoliberales que actúan como grilla de inteligibilidad del rol de la familia en la intervención sanitaria y que realzan un modo de subjetivación hacia un homo economicus aislado, orientado a buscar el beneficio personal y en el que los intereses de las familias se subordinan a los intereses de la maquinaria económica.

\section{CONCLUSIONES}

Las políticas de salud, como cualquier otra práctica de significación, es el resultado de determinados recursos culturales y negociaciones activas. De este modo, la posibilidad de que establezcan una mirada objetiva de los problemas y sujetos a los que atiende está puesta en discusión, sobre todo si uno de los principales recursos utilizados por la política es la metáfora, que, desde su formación como tropo del lenguaje, aleja al discurso de su propia transparencia. En este trabajo hemos evidenciado algunas modalidades de representación de la maternidad en el discurso estatal a través del análisis de cuatro metáforas — contenedor, libro, animal y empresa - que, cabe destacar, prueba los hallazgos encontrados en el campo de la lingüística cognitiva, que indica que los textos hacen uso de ella de forma automática, relevando asociaciones y correspondencias sedimentadas respecto a la maternidad y a las madres que resultan significativas y que comportan lo que podríamos llamar el «sentido común» de la política, al punto que esta producción lingüística es comprendida y compartida por cualquier lector. 
A las viejas significaciones organizadas al ya conocido «maternalismo» (Cosse, 2008; Flores y Tena, 2014) se ensamblan nuevas. Por un lado, se construye una imagen de la maternidad hermética y anclada a una supuesta naturaleza animal en que la díada se reactualiza como histórico objeto de intervención de la salud pública chilena. Esta dupla naturalizada, sin mediaciones de ningún tipo, es para Zárate y Godoy (2011) una «unidad ideológica» que afianza la maternidad como una función social y biológica que excluye al padre ${ }^{2}$ y una forma de idealizar la maternidad ocultando las condiciones de desigualdad en que se ejerce, así como a las mujeres como sujetos autónomos, con sus propias necesidades en salud y deseos, sugiriendo inclusive la subordinación de la salud de las mujeres a la salud del niño/a. Esto es un obstáculo para los logros de los objetivos de equidad, dado que se pasa por alto las dificultades de las mujeres para alcanzar estos ideales normativos, posibilitar negociaciones de las identidades maternas o para incorporar y darle significado a las experiencias novedosas o de resistencias.

Esta imagen de fusión complementaria bloquea los conflictos de intereses y necesidades entre las mujeres y los niños/as ya que, como señala Rosen (2018), las instituciones de salud llevan a cabo un hacer como si estos fueran naturalmente los mismos y/o complementarios. Así, se rechaza aquello que dice promover - la participación activa de los padres y las comunidades en las tareas de cuidados. Podemos decir entonces que emerge una modalidad de gubernamentalidad (Foucault, 2008) particular, en la que los trabajos de cuidado maternos se mantienen como actividad central de la producción social de la salud, pero a la vez son objeto de nuevas modulaciones expertas que atienden a los elementos capilares de relación entre madre y criatura, entre ellos, el control de las emociones o el lenguaje — como apreciamos en la metáfora del libro- para la formación de sujetos económicos determinados por las demandas de la sociedad del conocimiento, bajo la grilla de la ideología del capital humano. ${ }^{3}$ El sistema de salud, de esta manera, incorpora nuevas demandas que trascienden los polos de salud/enfermedad, ampliando su jurisdicción de forma dramática.

\footnotetext{
${ }^{2}$ Cabe destacar que, por motivos de espacio, no introducimos las expresiones metafóricas con las cuales se representa al padre y la paternidad. La más utilizada es la «paternidad activa», que se representa como resultado de procesos de aprendizaje. De esta forma, vemos que el padre es más remoto que la madre y que la correspondencia entre maternidad/naturaleza y paternidad/cultura es una matriz de pensamiento que cruza los objetivos del sistema.

${ }^{3}$ Parte de las intervenciones profesionales tienen que ver con la promoción de la lectura en las clases populares y lo que podríamos llamar el capital lingüístico
} 


\section{FINANCIACIÓN}

El presente trabajo fue parte del proyecto FONDECYT Iniciación $n^{\circ}$ 11160731, Ministerio de Ciencia y Tecnología/Chile: «Tecnologías de gobierno en la primera infancia y su cuidado: Un análisis discursivo del sistema protección social Chile Crece Contigo». 


\section{BIBLIOGRAFÍA}

ArteagA, Catalina; IÑIgO, Isidora. Políticas sociales, modelo de desarrollo y subjetividad de grupos vulnerables en Chile. En: Revista del CLAD Reforma y Democracia. 2015, vol. 61, pp. 209-234.

Austin, John. Cómo hacer cosas con palabras. Palabras y cosas. Barcelona: Paidós, 1991.

BERRI, Marina; BREgANT, Lucía. Identificación de metonimias y metáforas: cuestiones metodológicas. En: Lenguaje. 2015, vol. 43, núm. 2, pp. 219-245.

Blumer, Herbert. El interaccionismo simbólico. Perspectiva y método. Madrid: Hora, 1982.

CALLEJO, Javier. Lo que callar y discurso quieren decir para la sociología empírica. En: Cinta Moebio. 2019, vol. 65, pp. 194-208.

CÁrCAmo, Hernán. Hermenéutica y análisis cualitativo. En: Cinta Moebio. 2005, vol. 23, pp. 204-216.

CARO, Pamela. Análisis del programa Chile Crece Contigo desde los ideales normativos y la noción de prueba. En: Revista de trabajo social. 2009, vol. 77, pp. 25-37.

CASADO, Elena. Cyborgs, nómadas, mestizas: astucias metafóricas de la praxis feminista. En: Gatti, G.; Martínez de Albeniz, I. (eds.). Las astucias de la identidad. Figuras, territorios y estrategias de lo social contemporáneo. Bilbao: Servicio Editorial de la Universidad del País Vasco, 1999, pp. 41-59.

CASTEL, Robert. El ascenso de las incertidumbres. Trabajo, protecciones, estatuto del individuo. México: Fondo de Cultura Económica, 2010.

CASTILlo, Patricia. Los saberes psicológicos en el neoliberalismo: el caso de las políticas sociales y la teoría del apego en Chile. En: Universitas Psychologica. 2016, vol. 14, núm. 4, pp. 1325-1338.

Contreras, José; Rojas, Vicki; Contreras, Lorena. (2015). Análisis de programas relacionados con la intervención de niños, niñas y adolescentes vulnerados en sus derechos: La realidad chilena. En: Psicoperspectivas. 2015, vol. 14, núm. 1, pp. 89102.

Cosse, Isabella. Del matrimonio a la pareja: continuidades y rupturas en el modelo conyugal en buenos aires (1960-1975). En: Anuario IEHS. 2008, vol. 23, pp. 431458. 
Flores, Roberta; TenA, Olivia. Maternalismo y discursos feministas latinoamericanos sobre el trabajo de cuidados: un tejido en tensión. En: Íconos. 2014, núm. 50, pp. 2742.

FouCAULT, Michel. Seguridad, territorio y población. Madrid: Siglo XXI, 2008.

Fuentes, Maria; Brembeck, Helene. The construction and navigation of riskscapes in public health advice and mothers' accounts of weaning. En: Health, Risk \& Society. 2019, vol. 21, núm. 5-6, pp. 227-245.

GARRETÓN, Manuel. Neoliberalismo corregido y progresismo limitado: los gobiernos de la concertación en Chile, 1990-2010. Santiago: Arcis, 2012.

GonZÁlez de ReQuena, Juan. Procesos y construcciones: Un análisis de contenido de las metáforas en dos revistas de Psicología. En: Psicoperspectivas. 2014, vol. 13, núm. 2, pp. 174-185.

HARDY, Clarisa. Sistema de protección social en Chile: Red protege 2006-2010. Un Enfoque de derechos. En: Coloquio Internacional de Seguridad Social: Protección social, inclusión y equidad, 30 y 31 de agosto del 2011. [Consulta: 9 de marzo de 2020].

IMAZ, Elixabete. Mujeres gestantes, madres en gestación. Metáforas de un cuerpo fronterizo. En: Política y Sociedad. 2001, vol. 36, pp. 97-11.

ÍÑIGUEZ, Lupiciño. Análisis del discurso. Manual para las ciencias sociales. Barcelona: EDIUOC, 2006.

LAKOFF, George; Johnson, Mark. Metáforas de la vida cotidiana. Madrid: Cátedra, 1998.

LizCANo, Emmanuel. Metáforas que nos piensan. Sobre ciencia, democracia y otras poderosas ficciones. Madrid: Traficante de sueños, 2006.

MAGAÑA, Irene, et al. Diversidad familiar, relaciones de género y producción de cuidados en salud en el modelo de salud familiar: análisis de caso en un Cesfam de la región metropolitana, Chile. En: Terapia psicológica. 2011, vol. 29, núm. 1, pp. 33-42.

PAURA, Vilma; ZiBBECHI, Carla. Los programas de «Combate a la pobreza» en las agendas de gobierno y de estudio. Condiciones de producción académica, paradigmas argumentativos y revisiones conceptuales. En: POSTdata. 2018, vol. 23, núm. 2, p. 379-419.

Pragglejaz Group. MIP: A Method for Identifying Metaphorically Used Words. En: Discourse, Metaphor and Symbol. 2007, vol. 22, núm. 1, pp. 1-39. 
Rosen, Rachel; Twamley, Katherine. Feminism and the politics of childhood. Friends or foes. London: University College London, 2018.

Solimano, Andrés. Capitalismo a la chilena. Santiago: Catalonia, 2018.

VANDENDORPE, Christian. Las metáforas de la lectura en Del papiro al hipertexto. Ensayo sobre las mutaciones del texto y la lectura. México: Fondo de Cultura Económica, 1999.

ZÁRATE, María Soledad; Godoy, Lorena. Madres y niños en las políticas del Servicio Nacional de Salud de Chile (1952-1964). En: História, Ciências, Saúde-Manguinhos. 2011, núm. 18, pp. 131-151. 